\title{
A Survey of Vibration Energy Harvesters for Wireless Sensor Networks WANG Dong Ping ${ }^{1, a}$, YAN Zhen ${ }^{2, b}$ \\ ${ }^{1}$ Department of modern science \& technology, Agricultural University of Hebei, Hebei, China \\ ${ }^{2}$ Mechanical \& electrical engineering college, Agricultural University of Hebei, Hebei, China \\ awdpymh@126.com, bqceftgh@126.com
}

Keywords: vibration energy harvester; wireless sensor networks; energy harvesting

\begin{abstract}
With the fast developments of small dimension, low cost and low power circuit and sensor, energy supply is becoming a big barrier to wireless sensor networks applications in engineering, and the vibration energy harvester which not only gathering circumstance energy, but also lessening machine harm is an effective solving way. Framework of energy harvesting based wireless sensors networks is designed. And Geometric model, structural parameters, output results and technical characteristics of various vibration energy harvesters are particular introduced in recent three years. Finally future research needs and challenges of vibration energy harvesters for wireless sensor networks are simple analyzed.
\end{abstract}

\section{Introduction}

With the development of integrate circuit and micro electro mechanical systems, studying of circuit and sensor of small dimension and low cost and low energy consumption have obtained the high evolving, so the great wireless sensor networks(WSN) with thousands of nodes are formed which have widely application prospect for the fields of building health supervise, circumstance inspect, commonality safety and military affairs. However application of WSN is restricted because energy supply device cell has the flaw of the big dimension, limited life and replacement. So, the other energy sources should be exploited to supply the cell energy and even to replace the cell.

Solar energy, heat energy and vibration energy are prevailing energy sources in environment. Solar battery can convert the solar energy to electrical energy, but it only exits the circumstance of light. Thermoelectrical micro power source may convert the heat energy to electrical energy, but the way must exit the environment of temperature grades. Since vibration energy of environment exit anywhere in circumstance which including machine in plant, bridge, ship and car etc various equipment, and vibration in ambience sometimes can bring harm and short using life to machine. So, vibration energy is converted to recycle electric energy in which not only gathering energy, but also lessening machine harm and reducing the engine maintenance cost. At present, the vibration energy harvester has become the hotspot of power supply in WSN.

\section{Framework of energy harvesting based wireless sensors networks}

Since the acquired vibration energy is quite limited, the energy harvesting device should be designed with high efficiency, which converts, collects, and stores the energy to power the sensor networks as needed. As shown in Fig1, an energy harvesting WSN has a complicated framework including an energy harvesting module with harvesting device unit and energy harvesting circuit unit and power switching and power management model integrate within the WSN to establish a congruent interface with the energy harvesting module.

The harvesting device is the primary unit which converting vibration energy in circumstance to the electrical energy adopting vibration pick-up by electromagnetic or static or piezoelectric equipment. 


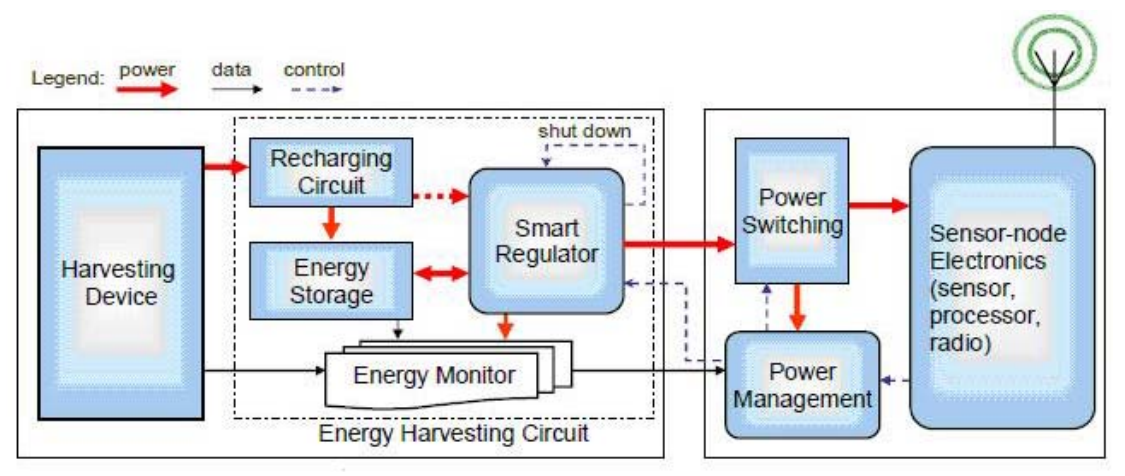

Energy Harvesting Module Wireless Sensor Networks

Figure 1 General structure of an energy harvesting module and compatible wireless sensor networks

In the energy harvesting circuit board, the recharging circuit, which is a condition circuit for energy storage device, has at least two functions. One is rectification converting AC voltage from harvesting device into DC voltage implemented by a diode bridge. The other function is to match the charging profile of the energy storage device which rechargeable batteries and ultracapacitors are commonly used. The smart regulator is the key component with function of adjusting the output power from the board to the WSN. The energy monitor tracks the available energy from environment, as well as the state of the energy storage device.

The power switching is to turn on or off various components of WSN as the amount of environmental availability varies. With the built-in power management algorithm, the power management module can make decision to turn on or off the power switching and control the smart regulator.

\section{Research evolving of vibration energy harvesters for self-powered wireless sensor networks}

Although the energy harvesting techniques are still in a development stage, some designs for WSN have been proposed in recent year. Following some studying and application of vibration energy harvesters for WSN from 2007 to 2009 are simply introduced.

Lei Wang and F. G. Yuan (2007) deigned a working prototype of a new class of vibration energy harvester based on magnetomechanical coupling using Magnetostrictive material (MsM), and the MsM energy harvester contains a transversely annealed Metglas 2605SC laminated beam and an electronic circuit on a PCB for interfacing with wireless sensors. The figure 2 show the Prototype of MsM energy harvesting device. According to the experiment results, the maximum output power and power density on a resistive load can reach $200 \mathrm{~W}$ and $900 \mathrm{~W} / \mathrm{cm} 3$ in low frequency, respectively. For practical usage, the average power and power density during charging the ultracapacitor can achieve $576 \mathrm{~W}$ and $606 \mathrm{~W} / \mathrm{cm} 3$ in high frequency, which are much higher than those of most piezo-based harvesters. However due to rapid quenching, the thickness of used in this study is limited to $18 \mathrm{~m}$, but thicker Metglas can reduce the load transfer loss and further enhance the output performance. So, how to enhance the thickness of standard Metglas ribbons need to further study[1].

R Torah and P Glynne-Jones etc(2008) designed an electromagnetic generator naming mk3 that has a volume of only $150 \mathrm{~mm} 3$ but produces sufficient electrical energy, from a very low-vibration source of $0.2 \mathrm{~m} / \mathrm{s} 2$, to power the entire microcontroller subsystem with no additional battery or power supply. The structure is shown in figure 3.

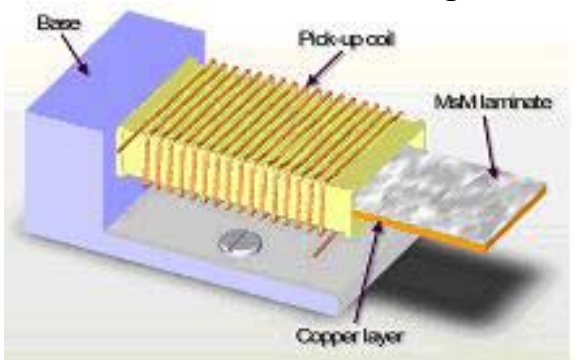

Figure 2 Prototype of MsM energy harvesting device

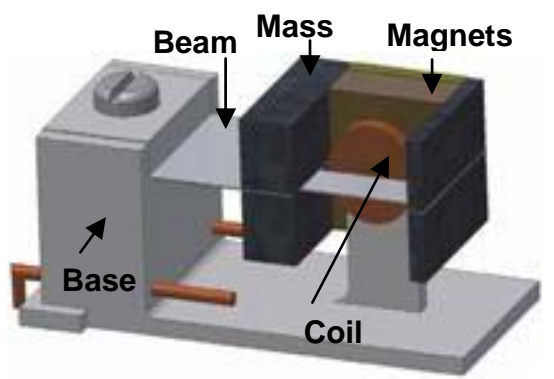

Figure $3 \mathrm{mk} 3$ generator design 
And the entire sensor system was tested and then demonstrated on both an air compressor and an office air conditioner unit in which transmitting a peak acceleration value every $3 \mathrm{~s}$ in both cases. Both applications had resonant peaks between 40 and $50 \mathrm{~Hz}$ with acceleration levels between 0.85 and $1.7 \mathrm{~m} / \mathrm{s} 2$. The WSN was operated continuously for $10 \mathrm{~h}$ and transmitted data throughout this time. Sufficient power is available for the transmitter to transmit data to a receiver module at a range of $20 \mathrm{~m}[2]$.

Future work of authors will consider the integration of data processing into the WSN and alternatively the transmission of larger amounts of data so that additional processing (e.g. a fast Fourier transform) can be performed at the receiver, thus improving the functionality of the system.

$\mathrm{Wu}$ yin-feng and Wan jiang-wen ect. (2008) brought forward a new electro-magnetic modeling method of deformed alternating direction implicit method - finite difference time domain (DADI-FDTD) which solving the difficulty of modeling radio frequency micro electro mechanical system (RF- MEMS) device for electromagnetic analysis, especially with small feature size and movable structures. The dynamic equation of motion is dispersed in time domain using finite difference methods (FDM). Numerical dispersion is discussed in the three-dimensional ADI-FDTD method. A new algorithm, dielectric interpolations, is presented, and it is capable of solving the conflict of the time and special steps between the mechanical portion and electromagnetic (EM) portion. And it is able to implement the EM modeling in time domain of complex three-dimensional structures. Simulation results demonstrate the feasibility of the method. But model only exit lab studying stage at present[3].

Wenzhi yu and Wenzhong yuan ect (2008) presented a new mathematical model of the vibration-based piezoelectric generator with the proof mass supported by a cantilever which is more accurate than Roundy's model. According to such characters as large transient power and small average power of the typical WSN,the power self-regulation circuit of the piezoelectric multi-shaker micro-generator is proposed. When the electricity in the super capacitor of the main circuit is enough to power the WSN,the main circuit is opened by the ancillary return circuit and the node starts to measure and transfer the results to the monitoring center. Final circuitry board and practical encapsulation circuit are showed in figure 4[4].

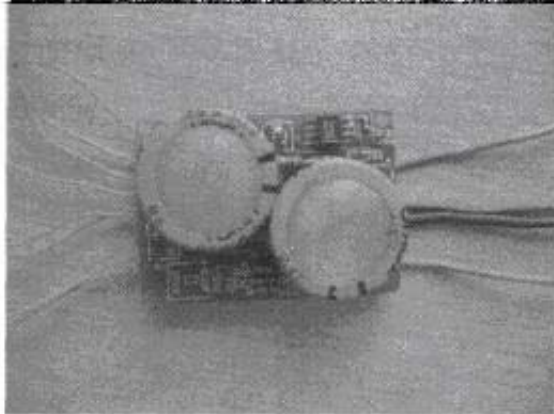

Figure 4 circuitry board and practical encapsulation circuit

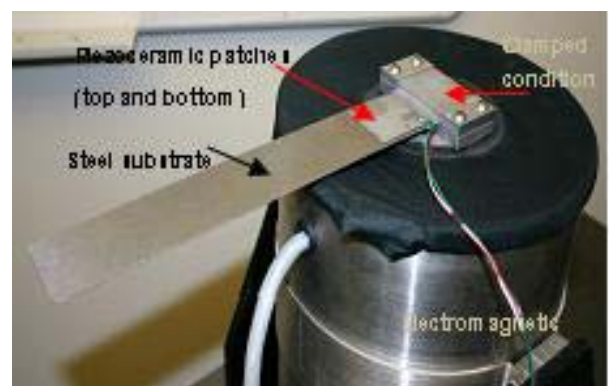

Figure 5 Cantilevered beam with piezoceramic patches mounted in bimorph

Kevin Farinholt and Stuart Taylor etc(2009) combine piezoelectric and thermoelectric transducers to harvest energy from vibrational and thermal sources commonly available on bridges, providing a more robust solution that does not rely on a single energy source. In piezoelectric energy harvester the piezoceramic lead zirconate titanate (PZT) was used as the electromechanical transducer. The length of the Steel substrate was used to tune the energy harvester's natural frequency to match one of the fundamental frequencies observed in the vibration response of the bridge. Once the energy harvesters had been tuned they were characterized with harmonic and multi-frequency base excitations through the electromagnetic shaker shown in Figure 5. The multi-frequency source was a filtered form of the bridge data that had been scaled by the shaker's inherent transfer function. Nevertheless, there still need to explore of insuring the coordinated work with the two kinds of energy harvester[5].

yang tao and qi hong-dong etc (2009) designed a Cantilever-type piezoelectric vibration transducers which is used in WSN nodes. Based on Taguchi robust design and genetic algorithm, an optimization method was developed with average power output as the robust optimization objectives. 
The energy collection circuit was designed using a tantalum capacitor and a zener diode . Through testing the prototypes with optimized parameters, the transducer characteristics were obtained. Experimental results showed that the generator could completely meet the requirements of sensor nodes with a power consumption of $50 \mu \mathrm{W}$. Structure simple and machining easy is the virtue of device[6].

\section{Future research needs and challenges}

While large-scale alternative sources such as wind turbines and solar cells which have made the transition from research to commercial products, energy harvesting for self-powered WSN is still in its infancy. Also, there is no clearly defined design process to develop such energy harvesting for self-powered WSN. Therefore, some future research areas for energy harvesting will be outlined in order to transition the current state-of-the-art to full-scale deployment in the current practice of WSN.

As identified, the major limitations in the field of energy harvesting revolve around the fact that the energy generated by harvesting devices is far too small to directly power most electronics. Therefore, the efficient and innovative methods of storing electric energy are the key technologies that will allow energy harvesting to become a source of power for electronics and wireless sensors.

Reliability is an essential requirement for any energy sources. Because many vibration-based harvesters are designed to operate at their resonances, the systems will be inherently unstable after the long operation cycles. Also, any energy sources for field use should be able to withstand harsh environmental conditions. The reliability and robustness must be proved before the energy harvesting techniques can be used in practice.

The goal of maximizing the amount of the harvested energy involves several factors, including electronics optimization, characterization of the available ambient energy, selection and configuration of energy harvesting materials, integration with storage mechanisms, along with the power-optimization and power-awareness design. Few studies have addressed these issues in an integrated manner from the multidisciplinary engineering perspective. Finally, it has been identified that, although several energy harvesting devices are developed and fabricated as a prototype, the performance of these techniques in real operational environments needs to be verified and validated.

\section{References}

[1] Lei Wang and F. G. Yuan: Energy Harvesting By Magnetostrictive Material (Msm) for Powering Wireless Sensors in SHM, Spie Smart Structures And Materials \& Node And Health Monitoring(2007), p. 18-22

[2] R Torah et al: Self-Powered Autonomous Wireless Sensor Node Using Vibration Energy Harvesting, Measurement Science And Technology(2008), p. 19-27

[3] Wu Yin-fen, Wan Jiang-we, Feng Ren-jia: Electromagnetic Modeling of Micro Sensor Node for Wireless Sensor Networ, Journal of Beijing University of Posts and Telecommunication, Vol.31 No.2(2008), p.10-14

[4] Wen Zhi-yu, Wen Zhong-quan, He Xue-feng, Liao Hai-yang, Liu Hai-tao: Vibration-based Piezoelectric Generator and Its Application in the Wireless Sensor Node, Chinese Journal of Mechanical Engineering, Vol 44, No.11(2008), p 75-79

[5] Kevin Farinholt et al: Energy Harvesting And Wireless Energy Transmission For Embedded Sensor Nodes, Active and Passive Smart Structures and Integrated Systems, Vol. 7288(2009)

[6] Yang Tao,Qi Hong-dong, Feng Yue-hui, Zeng Yong: A Piezoelectric Vibration Generator Used for Powering Wireless Sensor Network Node, Piezoelectectrics \& Acoustooptic, Vol .31, No.4(2009), p 510-513 